\title{
Influencia del pH en la adsorción de Cd(II) empleando estiércol de ganado vacuno
}

\section{Influence of $\mathrm{pH}$ on the absorption of $\mathrm{Cd}(\mathrm{II})$ using cattle manure}

\author{
'Fuentes López, W.S.; Bendezú Roca, Y.; Rosado Baldeón, R.Y.; \\ Camarena Valenzuela, M.A.; De La Cruz Ccanto, E. \\ Facultad de Ingeniería Química / Universidad Nacional del Centro del Perú \\ Email:wfuentes@uncp.edu.pe
}

\section{Resumen}

La presencia del cadmio Cd(II) en las aguas residuales domésticas e industriales, es propia del desarrollo industrial y crecimiento poblacional, su toxicidad, su efecto cancerígeno y su naturaleza bioacumulable son peligrosas para la salud. Se ha elegido el estiércol de ganado vacuno como adsorbente para evaluar su capacidad de adsorción de Cd(II). De soluciones preparadas a diferentes concentraciones donde el $\mathrm{pH}$ y el tiempo de contacto son las variables críticas del proceso. Al caracterizar el estiércol de ganado vacuno, la mayoría a los grupos funcionales corresponden a la pectina, celulosa, hemicelulosa y lignina, con gran capacidad para fijar iones de $\mathrm{Cd}(\mathrm{II})$, el análisis MEB, muestra indicios de formación de poros, el microanálisis elemental EDX muestra que está compuesta por $48 \%$ de carbono facilitando que los iones de Cd(II) se unan a las superficies del carbono, el análisis CIC indica que las superficies están cargados negativamente, atrayendo por fuerzas electrostáticas a los iones del Cd(II). La dosificación del estiércol de ganado vacuno fue de $0.0023 \mathrm{~g} / \mathrm{mL}$ alcanzando una remoción alrededor del $90 \%$, haciendo innecesario un incremento de adsorbente. El pH óptimo para la adsorción de $\mathrm{Cd}(\mathrm{II})$ fue de 3.5 , indicando que en un medio ácido se logra una desprotonación de los grupos carboxílicos, fenólicos y la mayoría de los grupos funcionales del estiércol, con uno y dos átomos de oxígeno cargados negativamente que actúan como agentes acomplejantes, que atraen a los iones de Cd(II). Finalmente, con $1 \mathrm{~g}$ de adsorbente se puede adsorber $2.3726 \mathrm{mg}$ de $\mathrm{Cd}(\mathrm{II})$ de soluciones acuosas.

Palabras Clave: aguas residuales, estiercol de ganado, grupos funcionales, adsorción, iones de Cd(II)

\begin{abstract}
The presence of cadmium Cd (II) in domestic and industrial wastewater is typical of industrial development and population growth. Its toxicity, its carcinogenic effect and its bioaccumulative nature are dangerous for health. Cattle manure has been chosen as an adsorbent to evaluate its capacity to adsorb Cd (II), from solutions prepared at different concentrations, where $\mathrm{pH}$ and contact time are the critical variables of the process. When characterizing cattle manure, most of the functional groups correspond to pectin, cellulose, hemicellulose and lignin, with great capacity to fix ions of Cd (II). The MEB analysis shows signs of pore formation, elemental EDX microanalysis shows that it is composed of $48 \%$ of carbon facilitating that the ions of $\mathrm{Cd}$ (II) are attached to the carbon surfaces, the CIC analysis indicates that the surfaces are negatively charged, attracting the ions of the $\mathrm{Cd}$ (II) by electrostatic forces. The dosage of cattle manure was $0.0023 \mathrm{~g} / \mathrm{mL}$ reaching a clearance of around $90 \%$, making unnecessary an increase in adsorbent. The optimal pH for adsorption of Cd (II) was 3.5 indicating that in an acidic medium a deprotonation of the carboxylic, phenolic and most functional groups of the manure is achieved, with one and two charged oxygen atoms negatively acting as complexing agents, which attract the Cd (II) ions. Finally, with $2 \mathrm{~g}$ of adsorbent, $2.3726 \mathrm{mg}$ of $\mathrm{Cd}$ (II) of aqueous solutions can be adsorbed.
\end{abstract}

Keywords: wastewater, cattle manure, functional groups, adsorption, cadmium(II) ions 


\section{Introducción}

El desarrollo industrial tiene hace uso de una variedad de recursos naturales donde el agua es imprescindible, el agua usada con o sin tratamiento, es vertida a los cauces mayores, lo que conlleva a que los ambientes acuáticos alcancen concentraciones de contaminantes que exceden los estándares establecidos para la calidad del agua. La calidad de las cuencas hidrográficas (Rosas, 2001), en las últimas décadas, se ha deteriorado por la presencia de metales pesados, debido al crecimiento de las poblaciones ribereñas y la industrialización. La presencia de Sb, As, Cd, Cu, Cr, Hg, Ni, Pb y Zn se distribuyen en el agua y en los sedimentos, así como hay biodisponibilidad en el medio acuático. La detección de la presencia de los elementos $\mathrm{Sb}, \mathrm{Cd}, \mathrm{Cu}, \mathrm{Cr}, \mathrm{Ni}, \mathrm{Pb}$ se hace por espectrometría de masas (ICP-MS) ELAN 6000; del mismo modo, que los parámetros físico-químicos y los carbonatos, materia orgánica y silicatos; con el fin de correlacionar entres sus contenidos en el agua y los de metales pesados en los sedimentos. En cuanto a los metales pesados, el cadmio es muy empleado en galvanoplastía, contra la corrosión, en la estabilización de los plásticos, etc. Es necesario la eliminación del cadmio o por lo menos minimizar su presencia a niveles muy por debajo de los límites de tolerancia, debido a su elevada toxicidad y por ser considerado un elemento cancerígeno; además, puede afectar a los genes, causar hiperglucemia, debilitar el sistema inmunológico y producir anemia por su interferencia con el metabolismo del hierro.

En las actividades minero-metalúrgicas, las aguas superficiales y las subterráneas son la fuente principal de la presencia del ion cadmio Cd(II), el cual es consumido directamente del agua o indirectamente a través de los cultivos; además, es bioacumulable. Para la eliminación del Cd(II) de soluciones preparadas a diferentes concentraciones; la revisión de los antecedentes nos indica elegir, la adsorción como método y el estiércol de ganado vacuno como adsorbente, por su efectividad que puede alcanzar hasta un $90 \%$ de remoción.

El uso del estiércol como adsorbente es reciente, por lo que es necesario estudiar las variables más significativas que influyen en la capacidad de adsorción de soluciones acuosas. Se ha identificado el pH y el tiempo de contacto como variables significativas; con el objetivo de evaluar la influencia del pH y el tiempo de contacto, en la adsorción del Cd(II) de soluciones acuosas, empleando estiércol de ganado vacuno recolectado de la localidad de Sicaya. El estiércol se ha pulverizado y secado para caracterizarlo mediante FTIR, microscopía electrónica de barrido (MEB) y fisisorción por adsorción de N2, con la finalidad de determinar el área superficial y la distribución de poros; seguidamente se han realizado pruebas preliminares; primero para determinar el pH adecuado donde se logra una mejor adsorción del Cd(II) de soluciones acuosas y, luego, determinar el tiempo de contacto donde se alcanza una máxima adsorción, empleando el estiércol de ganado vacuno previamente preparado para tal fin. Los resultados obtenidos son alentadores.

\section{Materiales y método}

La investigación es aplicada a un nivel explicativo, se pretende explicar los efectos de la variación del $\mathrm{pH}$ y el tiempo de contacto, en la remoción del Cd(II) de soluciones acuosas preparadas a diferentes concentraciones; para ello, empleamos el método experimental, con un diseño, antes y después de modificar el $\mathrm{pH}$ y el tiempo de contacto. En este caso, se ha elegido como población a la solución preparada de nitrato de cadmio - Solución de Cd(NO3)2 a $1000 \mathrm{mg} / \mathrm{L}$ y como muestra unos 150 $\mathrm{mL}$ de solución patrón de $\mathrm{Cd}(\mathrm{NO} 3) 2$, a $1000 \mathrm{mg} / \mathrm{L}$ de marca Merck. Además, se emplea el agua destilada, trozos de hielo y el estiércol de ganado vacuno finamente pulverizado.

\section{Método}

Primero, se realizó la preparación del adsorbente a base del estiércol de ganado vacuno, eligiéndose el punto de muestreo, realizando luego la recolección, limpieza, secado, molido y tamizado. Después se determina la humedad y las características fisicoquímicas, que nos indicarán los grupos funcionales y las particularidades superficiales del adsorbente. Finalmente, se realiza la interacción con la solución preparada de Cd(II) y se determina la adsorción.

\section{Muestreo y preparación del adsorbente.}

El muestreo del estiércol de ganado vacuno, se realizó en el barrio Santa Bárbara del distrito de Sicaya, como se aprecia en la Figura 1, ubicada en la margen derecha del río Mantaro, en la provincia de Huancayo.

La técnica de muestreo, del estiércol de ganado vacuno, fue en zigzag, como se observa en la Figura 2, tomándose 15 sub muestras de 750 g cada uno, acumulando $11.250 \mathrm{~kg}$. Se tomaron de una profundidad de $15 \mathrm{~cm}$. Las muestras extraídas se depositaron en bolsas de mica especiales y debidamente etiquetadas. Las sub muestras fueron secadas y molidas, manualmente para homogenizar y tomar una cantidad representativa, para ser preparada y caracterizada.

Figura 1

Vista satelital del lugar de muestreo, barrio Santa Bárbara-Sicaya-Huancayo-Junín

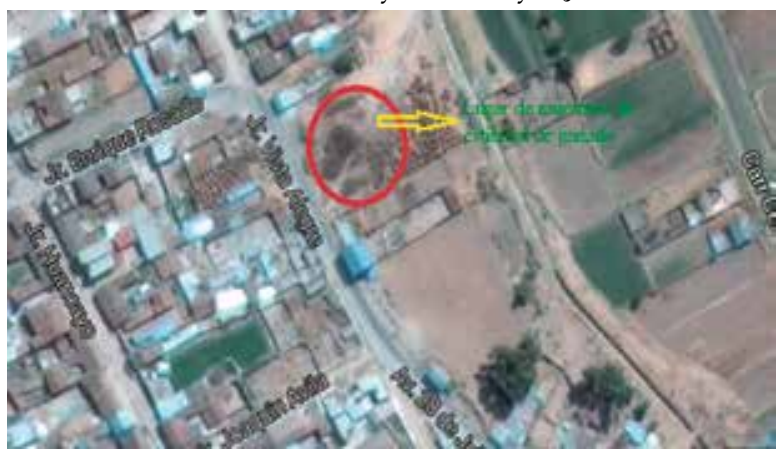

Fuente: Google Maps (2017) 


\section{Figura 2}

Muestreo del estiércol de ganado vacuno, barrio Santa Bárbara-Sicaya-Huancayo-Junín

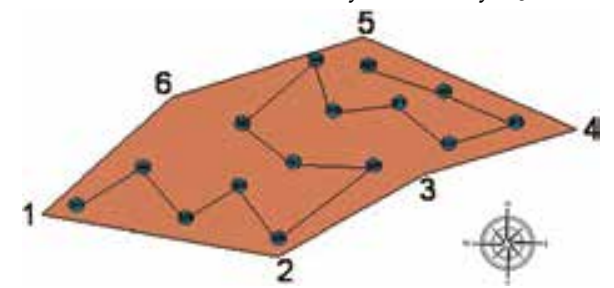

Fuente: Elaboración propia (2017)

Preparación del estiércol de ganado vacuno: selección, limpieza y secado.

A partir de la muestra homogenizada, se separaron las sustancias sólidas adheridas al estiércol de ganado vacuno, el peso de la muestra para ser secado fue 10.850 $\mathrm{kg}$, quedando al final un total de $7.250 \mathrm{~kg}$. El secado se realizó a temperatura ambiente, se trituró la muestra y se almacenó en tres bandejas, cada una con $750 \mathrm{~g}$ de muestra, cubiertos con papel aluminio, exponiéndolas al sol interrumpidamente por 6 horas durante 20 días, por las mañanas.

\section{Determinación del porcentaje de humedad.}

Después del secado se obtuvo un peso final de $501.2 \mathrm{~g}$, el cual se usó para determinar el porcentaje de humedad del estiércol de ganado vacuno, con la ecuación.

$$
\text { \% Humedad }=\frac{\left(\mathrm{P}_{\mathrm{i}}-\mathrm{P}_{\mathrm{f}}\right) * 100}{\mathrm{P}_{\mathrm{i}}}
$$

Donde:

$\mathrm{P}_{\mathrm{i}}$ : $\quad$ Peso inicial de estiércol de ganado vacuno antes del secado $(g)=750 \mathrm{~g}$.

$\mathrm{P}_{\mathrm{f}}$ : $\quad$ Peso final de estiércol de ganado vacuno después del secado $(g)=501.2 \mathrm{~g}$.

Siendo el \% Humedad $=33,17 \%$

\section{Molienda y tamizado.}

Para la molienda de la muestra seca, se utilizaron un molino manual y un mortero, el tamizado se hizo a malla -70 $(212 \mu \mathrm{m})$. Finalmente, el peso del adsorbente después del tamizado fue $2.650 \mathrm{~kg}$.

\section{Resultados}

La caracterización química, física y fisicoquímica del estiércol de ganado vacuno, arroja, según el tipo de análisis, los siguientes resultados:

\section{Análisis espectroscopía infrarroja por transformada de Fourier (FT-IR).}

La espectroscopía FT-IR, del estiércol de ganado vacuno, ha identificado señales asociados a los grupos funcionales, como de la pectina, celulosa, hemice- lulosa y lignina, los cuales se encuentran en el interior de las paredes celulares y los grupos funcionales como: carboxilo, amino e hidroxilo, que poseen sitios activos de unión capaces de secuestrar iones de Cd(II). Como se puede observar en la Figura 3, donde se muestran los espectros del infrarrojo del estiércol de ganado vacuno que lo tipifica como adsorbente.

Análisis morfológico por microscopía electrónica de barrido (MEB) y microanálisis elemental por espectroscopía por dispersión de rayos $X(E D X)$.

La caracterización morfológica del estiércol de ganado vacuno realizado por Microscopía Electrónica de Barrido - MEB que se acopla con la Espectroscopía por Dispersión de Rayos X - EDX, no indican la calidad del estiércol de ganado vacuno como adsorbente. En la Figura 4 y la Figura 5, se muestran dos de las varias micrografías MEB, del estiércol de ganado vacuno, con aumentos de: 50x y 10000x; en las cuales se observa que son heterogéneas, apreciándose al menos seis tipos de estructuras: (1) fragmentos de fibra, (2) bloques con hendiduras, (3) rejillas cuadriculares, (4) fragmentos minúsculos incrustados, (5) espirales heterogéneas y (6) bloques de rajaduras, todas estas distribuidas de manera aleatoria.

Figura 3

Espectros de FT-IR del estiércol de ganado vacuno (adsorbente)

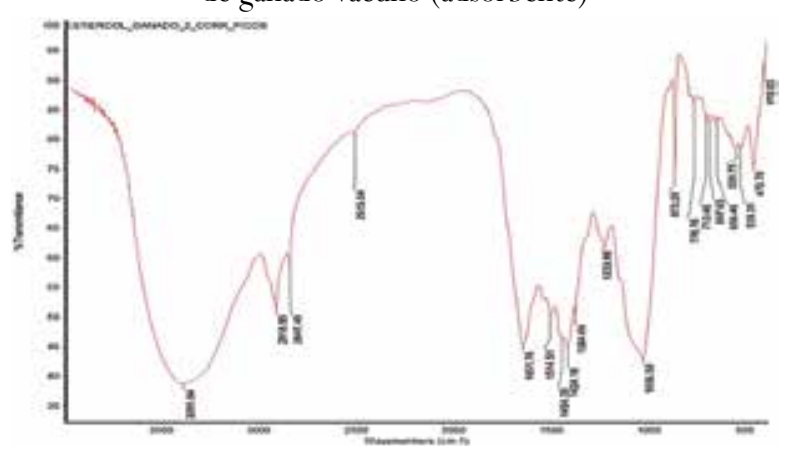

Fuente: Laboratorio de Recursos Analíticos y de Calibración FIQ UNICAMP-Sao Paulo Brasil (2017)

Figura 4

Micrografía del estiércol con aumento de 50x

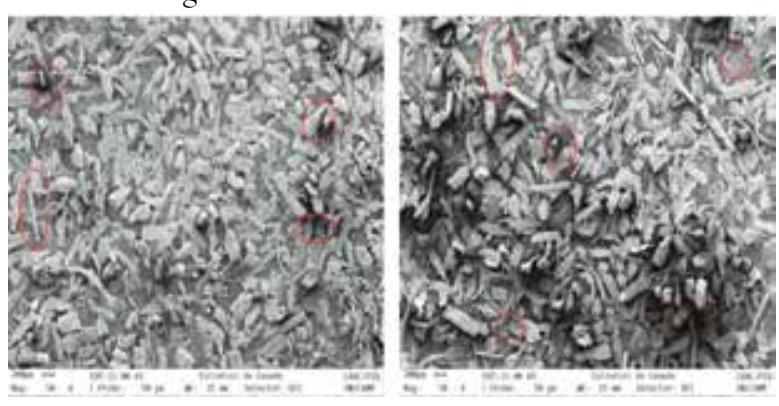

Fuente: Laboratorio de Recursos Analíticos y de Calibración FIQ UNICAMP-Sao Paulo Brasil (2017) 
Figura 5

Micrografía del estiércol con aumento de 10000x

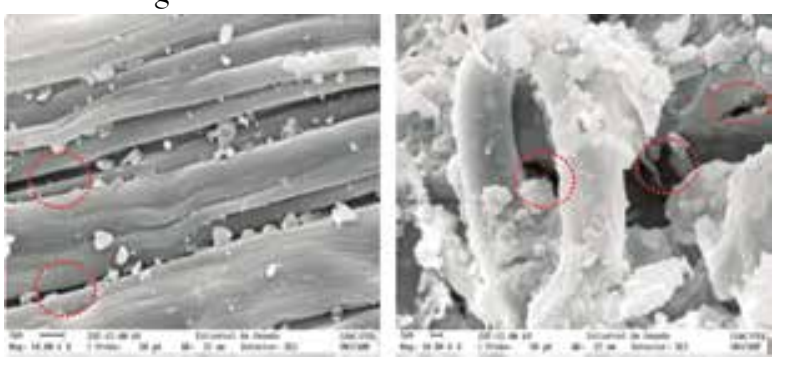

Fuente: Laboratorio de Recursos Analíticos y de Calibración FIQ UNICAMP-Sao Paulo Brasil (2017)

Por otro lado, en la Tabla 1 , se reúnen los resultados del microanálisis elemental por EDX, de la muestra de estiércol de ganado vacuno, expresados en 5 espectros diferentes, que en promedio alcanzan el $48 \%$ de $\mathrm{C}$, el $42 \%$ es $\mathrm{O}$ y otros elementos representan solo el $10 \%$.

Tabla 1

Microanálisis elemental por espectroscopia por dispersión de rayos $\mathrm{X}$, de la muestra de estiércol de ganado vacuno.

\begin{tabular}{|c|c|c|c|c|c|}
\hline \multirow{2}{*}{ 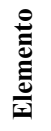 } & \multicolumn{5}{|c|}{ Estiércol de ganado vacuno } \\
\hline & $\begin{array}{c}\text { L1 } \\
\text { (\%) }\end{array}$ & $\begin{array}{c}\text { L2 } \\
\text { (\%) }\end{array}$ & $\begin{array}{c}\text { L3 } \\
(\%)\end{array}$ & $\begin{array}{c}\text { L4 } \\
\text { (\%) }\end{array}$ & $\begin{array}{l}\text { L5 } \\
\text { (\%) }\end{array}$ \\
\hline $\mathrm{C}$ & 51.94 & 46.13 & 46.60 & 46.91 & 48.89 \\
\hline $\mathrm{O}$ & 40.05 & 43.33 & 42.75 & 42.75 & 43.18 \\
\hline $\mathrm{Na}$ & - & 0.34 & - & - & - \\
\hline $\mathrm{Mg}$ & 0.40 & 0.69 & 0.63 & 0.53 & 0.48 \\
\hline $\mathrm{Al}$ & 0.25 & 0.30 & 0.27 & 0.34 & 0.20 \\
\hline $\mathrm{Si}$ & 1.95 & 2.16 & 2.07 & 2.31 & 1.87 \\
\hline $\mathrm{Cl}$ & 1.14 & 1.45 & 1.40 & 1.49 & 0.88 \\
\hline $\mathrm{K}$ & 2.25 & 3.09 & 3.15 & 3.17 & 2.16 \\
\hline $\mathrm{Ca}$ & 2.02 & 2.51 & 2.76 & 2.65 & 2.06 \\
\hline $\mathrm{Fe}$ & - & - & - & 0.35 & 0.28 \\
\hline
\end{tabular}

Fuente: Laboratorio de Recursos Analíticos y de Calibración FIQ UNICAMP-Sao Paulo Brasil (2017)

Análisis del área superficial específica y porosidad del estiércol de ganado vacuno.

El análisis textural del estiércol de ganado vacuno, fue obtenido mediante la isoterma de adsorción de N2 a temperatura $77 \mathrm{~K}$, para identificar el área superficial se utilizó la ecuación de Brunauer-Emmett-Teller - BET para un sistema sólido-gas; donde de acuerdo a su diámetro medio de poro (IUPAC-1997), (microsoporos < $2 \mathrm{~nm}$, mesosporos 2-50 nm, macroporos 50-100000 $\mathrm{nm})$, los del estiércol de ganado vacuno estarían en la clasificación de los mesoporos, datos que se muestran en la Tabla 2, donde se presentan los parámetros del análisis fisicoquímico del estiércol de ganado vacuno (adsorbente).
Tabla 2

Parámetros de análisis estructural del estiércol de ganado vacuno (adsorbente)

\begin{tabular}{cccc}
\hline $\begin{array}{c}\text { Área } \\
\text { superficial } \\
\text { específica BET } \\
\left(\mathbf{m}^{2} / \mathbf{g}\right)\end{array}$ & $\begin{array}{c}\text { Volumen de } \\
\text { poro }\left(\mathbf{c m}^{3} / \mathbf{g}\right)\end{array}$ & $\begin{array}{c}\text { Diámetro } \\
\text { medio de poro } \\
(\AA)\end{array}$ & $\begin{array}{c}\text { Diámetro } \\
\text { medio de poro } \\
(\mathbf{n m})\end{array}$ \\
\hline 1.6073 & 0.005642 & 109.452 & 10.9452 \\
\hline
\end{tabular}

Fuente: Laboratorio de Recursos Analíticos y de Calibración FIQ UNICAMP-Sao Paulo Brasil (2017)

Análisis de materia orgánica (M.O.) y capacidad de intercambio catiónico (C.I.C.).

El mayor contenido de la materia orgánica, del estiércol de ganado vacuno, es el carbono que está presente en todas sus formas. En la Tabla 3, se observa que alcanza un valor cercano al $4 \%$, superando al potasio, fósforo y nitrógeno, quienes contribuyen en la CIC del adsorbente.

Tabla 3

Porcentaje del contenido de materia orgánica

M.O. del estiércol de ganado vacuno (adsorbente)

\begin{tabular}{cccccccc}
\hline $\begin{array}{c}\mathbf{N}^{\circ} \\
\text { Lab. }\end{array}$ & Claves & pH & $\begin{array}{c}\text { C.E. } \\
(\mathbf{d S} / \mathbf{m})\end{array}$ & $\begin{array}{c}\text { M.O. } \\
(\mathbf{\%})\end{array}$ & $\begin{array}{c}\mathbf{N} \\
(\mathbf{\%})\end{array}$ & $\begin{array}{c}\mathbf{P}_{\mathbf{2}} \mathbf{O}_{\mathbf{5}} \\
(\mathbf{\%})\end{array}$ & $\begin{array}{c}\mathbf{K}_{\mathbf{2}} \mathbf{O} \\
(\mathbf{\%})\end{array}$ \\
\hline 13 & - & 8.02 & 9.20 & 3.91 & 2.34 & 1.40 & 3.48 \\
\hline
\end{tabular}

Fuente: Laboratorio de Análisis de Suelos, Plantas, Aguas y Fertilizantes de la Facultad de Agronomía de la UNALM (2017)

Donde:

$\mathrm{pH}$ : Potencial de hidrógeno.

C.E: Conductividad eléctrica.

M.O: Materia orgánica.

$\mathrm{N}$ : Nitrógeno.

P2O5: Óxido de fósforo (V).

K2O: Óxido de potasio.

La capacidad de intercambio catiónico (CIC), es la capacidad que tiene el adsorbente, para retener y liberar iones positivos, esta crece según el contenido de materia orgánica y arcillas, la misma que se mide en meq/100 g. En la Tabla 4, se registra 38,00 meq/100 g como la CIC del estiércol de ganado vacuno, indicando que las superficies de las moléculas están cargadas negativamente.

En el análisis de la capacidad de intercambio catiónico, del estiércol de ganado vacuno, se observó un 38.00 meq/100 $\mathrm{g}$, esto indica que las superficies de las moléculas están cargadas negativamente, los iones del Cd(II) son atraídos por estas superficies negativas por fuerzas electrostáticas.

Tabla 4

Capacidad de intercambio catiónico presente en el estiércol de ganado vacuno.

\begin{tabular}{ccccccc}
\hline $\begin{array}{c}\mathbf{N}^{\circ} \\
\text { Lab. }\end{array}$ & Claves & $\begin{array}{c}\mathrm{Ca} \\
(\%)\end{array}$ & $\begin{array}{c}\text { MgO } \\
(\%)\end{array}$ & $\begin{array}{c}\text { H.d. } \\
(\%)\end{array}$ & $\begin{array}{c}\text { Na } \\
(\%)\end{array}$ & $\begin{array}{c}\text { C.I.C. } \\
\mathbf{m e q} / \mathbf{1 0 0 g}\end{array}$ \\
\hline 13 & - & 4.97 & 1.28 & 7.03 & 0.23 & 38.00 \\
\hline
\end{tabular}

Fuente: Laboratorio de Análisis de Suelos, Plantas, Aguas y Fertilizantes de la Facultad de Agronomía de la UNALM (2017) 
Donde:

H.d: Humedad disponible

\section{Resultados de la dosificación de estiércol de ganado vacuno en la adsorción de Cd(II)}

Para determinar la dosificación, del adsorbente, se realizaron 10 pruebas, el volumen inicial de la solución preparada es $0.550 \mathrm{~L}$ y la concentración inicial (Ci) de Cd(II) presente en todas las muestras es $4,851 \mathrm{mg} / \mathrm{L}$ y las masas del adsorbente varían desde $0.0005 \mathrm{~g}$ hasta $0.0032 \mathrm{~g}$ por cada $\mathrm{mL}$ de solución, incrementándose $0.0003 \mathrm{~g}$ por muestra. La agitación fue de 90 minutos a 300 RPM.

Los resultados se pueden observar en la Tabla 5, donde se aprecia que el porcentaje de remoción $(\% \mathrm{R})$ correspondientes a la muestra CM-10, para la dosis de adsorbente $0.0032 \mathrm{~g} / \mathrm{mL}$, es la mayor, lo que nos indica que la adsorción es directamente proporcional a la dosis del adsorbente.

Tabla 5

Dosis de adsorbente, concentración inicial, concentración final en el equilibrio y porcentaje de remoción

\begin{tabular}{|c|c|c|c|c|}
\hline Código & $\begin{array}{c}\text { Dosis de } \\
\text { adsor- } \\
\text { bente (g/ } \\
\text { mL) }\end{array}$ & $\begin{array}{c}\text { Ci de } \\
\text { Cd(II) } \\
(\mathrm{mg} / \mathrm{L})\end{array}$ & $\begin{array}{l}\text { Cf de } \\
\text { Cd(II) } \\
(\mathrm{mg} / \mathrm{L})\end{array}$ & $\% R$ \\
\hline CM-11 & 0.0000 & 4.851 & 4.851 & 0 \\
\hline CM-01 & 0.0005 & 4.851 & 4.608 & 5.009 \\
\hline CM-02 & 0.0008 & 4.851 & 4.345 & 10.431 \\
\hline CM-03 & 0.0011 & 4.851 & 3.629 & 25.191 \\
\hline CM-04 & 0.0014 & 4.851 & 2.164 & 55.391 \\
\hline CM-05 & 0.0017 & 4.851 & 1.052 & 78.314 \\
\hline CM-06 & 0.0020 & 4.851 & 0.734 & 84.869 \\
\hline CM-07 & 0.0023 & 4.851 & 0.474 & 90.229 \\
\hline CM-08 & 0.0026 & 4.851 & 0.511 & 89.466 \\
\hline CM-09 & 0.0029 & 4.851 & 0.486 & 89.981 \\
\hline CM-10 & 0.0032 & 4.851 & 0.461 & 90.497 \\
\hline
\end{tabular}

Fuente: NSF Envirolab S.A.C (2016)

La dependencia de la dosis de estiércol de ganado vacuno, como adsorbente, con el porcentaje de remoción (\%) del Cd (II) se puede observar en la Figura 6 , donde al incrementar la dosis del adsorbente, el porcentaje de adsorción de $\mathrm{Cd}$ (II) se incrementa considerablemente, desde $5.0 \%$ de adsorción, para una dosis de $0.0005 \mathrm{~g} / \mathrm{mL}$, hasta superar el $90 \%$ para una dosis de $0.0023 \mathrm{~g} / \mathrm{mL}$ de la muestra CM-07, pero sin llegar al equilibrio.
Figura 6

Relación de la dosis de adsorbente $(\mathrm{g} / \mathrm{mL})$ con el porcentaje de remoción (\%)

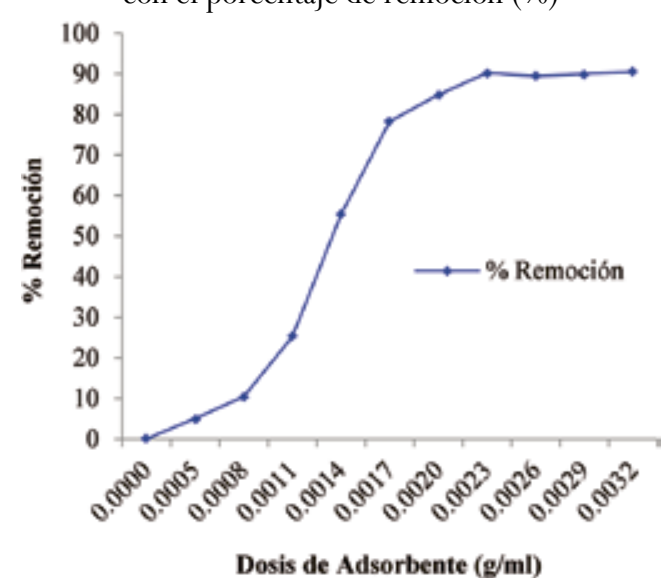

Fuente: Elaboración propia (2017).

A partir de la muestra CM-07 hay una tendencia a la estabilidad, alcanzando una máxima adsorción de iones de $\mathrm{Cd}(\mathrm{II})$ en la muestra CM-10, que permanece fluctuando en los alrededores de la vecindad de $90 \%$. Para los sucesivos experimentos se empleará la dosis de $0.0023 \mathrm{~g} / \mathrm{L}$ con el que se logró un alto porcentaje de adsorción, ya que un incremento del adsorbente no sería significativo, puesto que el estiércol de ganado vacuno tiende a secuestrar gran cantidad protones del medio, elevando en gran medida, el pH de la solución, lo que influiría la solubilidad del Cd(II).

\section{Resultados del efecto del pH en la adsorción de Cd(II) con estiércol de ganado vacuno.}

Se realizaron 10 pruebas, variando el $\mathrm{pH}$ desde 2.0 hasta 6.5 con un incremento de 0.5 , con un volumen inicial de $0.550 \mathrm{~L}$ de la solución preparada y 0.0023 $\mathrm{g} / \mathrm{mL}$ de masa del adsorbente; con agitación de 90 minutos a 300 RPM. En la Tabla 6, se observa que el mayor porcentaje de remoción de $\mathrm{Cd}(\mathrm{II})$ se logra a un $\mathrm{pH}$ de 3.5 que corresponde a la muestra CPH-M4 donde se alcanza un \%R de $94.94 \%$ (95\%).

El incremento del pH de 2.0 a 2.5 genera un aumento considerable en la remoción, llegando a $80.44 \%$ y, al pasar de 3.0 hasta 3.5, alcanza su máximo, aproximándose al $95 \%$ de remoción; se debe a que en un medio ácido se logra una desprotonación de los grupos funcionales que contiene el estiércol de ganado vacuno, presentándose en mayor porcentaje los grupos carboxílicos y fenólicos, los cuales se pueden desprotonar en iones carboxilato y fenoxido con uno y dos átomos de oxígeno respectivamente, cargándose negativamente y actuando como agentes acomplejantes, que atraen a los iones de $\mathrm{Cd}(\mathrm{II})$. Al incrementar el pH de 5.5 hasta 5.6 la remoción y adsorción disminuyen, según la Figura 7. 
Tabla 6

Dosis de adsorbente, concentración inicial y final de Cd(II), pH de la solución capacidad de adsorción y porcentaje de remoción

\begin{tabular}{cccccccc}
\hline Código & $\begin{array}{c}\text { Dosis de adsor- } \\
\text { bente (g/mL) }\end{array}$ & $\begin{array}{c}\text { Volumen de } \\
\text { la solución } \\
(\mathbf{L})\end{array}$ & $\begin{array}{c}\mathbf{p H} \text { de la } \\
\text { solución }\end{array}$ & $\begin{array}{c}\text { Ci de Cd(II) } \\
(\mathbf{m g} / \mathbf{L})\end{array}$ & $\begin{array}{c}\mathbf{C f} \mathbf{d e} \mathbf{C d}(\mathbf{I I}) \\
(\mathbf{m g} / \mathbf{L})\end{array}$ & $\begin{array}{c}\mathbf{q}_{\mathbf{e}} \\
(\mathbf{m g} / \mathbf{g})\end{array}$ & \%R \\
\hline CP-M0 & 0.0000 & 0.550 & 2.5 & 5.650 & 5.650 & - & 0 \\
CPH-M1 & 0.0023 & 0.550 & 2.0 & 5.650 & 4.732 & 0.3991304 & 16.25 \\
CPH-M2 & 0.0023 & 0.550 & 2.5 & 5.650 & 1.105 & 1.9760870 & 80.44 \\
CPH-M3 & 0.0023 & 0.550 & 3.0 & 5.650 & 0.365 & 2.2978261 & 93.54 \\
CPH-M4 & 0.0023 & 0.550 & 3.5 & 5.650 & 0.286 & 2.3321739 & 94.94 \\
CPH-M5 & 0.0023 & 0.550 & 4.0 & 5.650 & 0.331 & 2.3126087 & 94.14 \\
CPH-M6 & 0.0023 & 0.550 & 4.5 & 5.650 & 0.376 & 2.2930435 & 93.35 \\
CPH-M7 & 0.0023 & 0.550 & 5.0 & 5.650 & 0.362 & 2.2992754 & 93.60 \\
CPH-M8 & 0.0023 & 0.550 & 5.5 & 5.650 & 0.377 & 2.2926087 & 93.33 \\
CPH-M9 & 0.0023 & 0.550 & 6.0 & 5.650 & 0.540 & 2.2217391 & 90.44 \\
CPH-M10 & 0.0023 & 0.550 & 6.5 & 5.650 & 0.723 & 2.1421739 & 87.20 \\
\hline
\end{tabular}

Fuente: NSF Envirolab S.A.C (2017)

Figura 7

Relación del pH de la solución con el porcentaje de adsorción (\%) y la capacidad de adsorción qe $(\mathrm{mg} / \mathrm{g})$

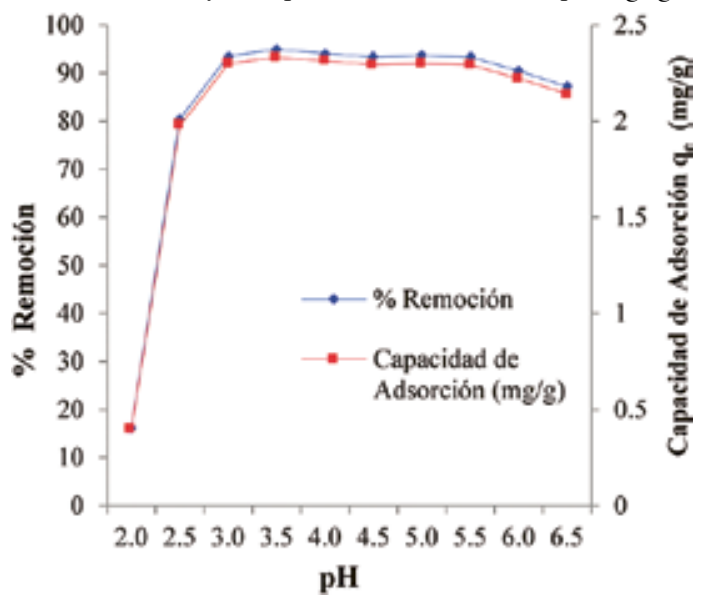

Fuente: Elaboración propia (2017)

Resultados de las isotermas de adsorción de Cd(II) empleando estiércol de ganado vacuno.

El estudio de las isotermas de adsorción de Cd(II), se hicieron con soluciones de $\mathrm{Cd}(\mathrm{II})$ a concentraciones de $1,2,4,5$ y $6 \mathrm{mg} / \mathrm{L}$. Se emplearon los valores determinados para el adsorbente la dosis de $0.0023 \mathrm{~g} /$ $\mathrm{mL}$, el volumen de la solución $0.450 \mathrm{~L}$, los tiempos de contacto de 15, 30, 60, 75 y 90 minutos con agitación a 300 RPM. La determinación de las concentraciones, inicial y final; se hicieron por Espectroscopía de Emisión Atómica de Plasma Acoplado Inductivamente (ICP-AES).

En la Tabla 7, se observa que el tiempo de contacto no influye significativamente en la capacidad de adsorción del estiércol de ganado. Asumiendo el tiempo de 90 minutos, como base para el estudio de las otras variables.

Así mismo, una isoterma de adsorción lineal del tipo C, indica que el adsorbato presenta mayor afinidad por el adsorbente que por el disolvente. La forma lineal de la isoterma indica que el soluto penetra en zonas inaccesibles al disolvente. La isoterma lineal del subgrupo 1 representa que la monocapa no ha sido completada por los adsorbatos.

La Figura 8, muestra el comportamiento lineal de las isotermas, para los tiempos de 15, 30, 60, 75 y 90 minutos, en el sistema sólido-líquido.

Figura 8

Isotermas de adsorción para los tiempos de contacto de 15, 30, 60, 75, 90 minutos.

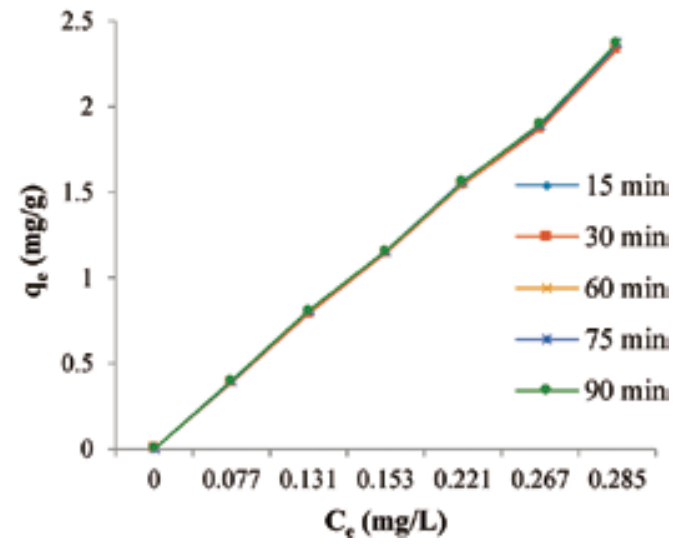

Fuente: Elaboración propia (2017) 


\section{Discusión}

Evaluar la capacidad del estiércol de ganado vacuno como adsorbente de $\mathrm{Cd}(\mathrm{II})$, requiere un adecuado muestreo, preparación y caracterización química, física y fisicoquímica para establecer las condiciones de las variables de trabajo. encontrándose, según los reportes del análisis por FT-IR, informa que la mayoría de los grupos funcionales corresponden a la pectina, celulosa, hemicelulosa y lignina, que tienen gran capacidad para fijar iones de Cd(II). En el análisis MEB, se observaron muchas estructuras con indicios de formación de poros, estos también favorecen la adsorción de Cd(II), en la misma línea, el microanálisis elemental por EDX, indica la presencia de carbono en un $48 \%$, favoreciendo la unión de los iones $\mathrm{Cd}$ (II) a las superficies del carbono, además que el área superficial específica y la porosidad se encuentran en el rango de los mesoporos, lo que facilita, más aún, la adsorción. La CIC alcanza un 38,00 meq/100 g, esto indica que las superficies están cargadas negativamente atrayendo por fuerzas electrostáticas a los iones del Cd(II).

Tabla 7

Concentración inicial, final y capacidad de adsorción, para 15, 30, 60, 75 y 90 minutos.

\begin{tabular}{|c|c|c|c|c|c|c|}
\hline Código & $\begin{array}{c}\text { Dosis de } \\
\text { adsor- } \\
\text { bente (g/ } \\
\mathrm{mL})\end{array}$ & $\begin{array}{l}\text { Volu- } \\
\text { men de } \\
\text { la solu- } \\
\text { ción (L) }\end{array}$ & $\begin{array}{l}\text { Tiempo } \\
\text { t (min) }\end{array}$ & $\begin{array}{c}\text { Ci de } \\
\mathrm{Cd}(\mathrm{II}) \\
(\mathrm{mg} / \mathrm{L})\end{array}$ & $\begin{array}{l}\text { Cf de } \\
\text { Cd(II) } \\
(\mathrm{mg} / \mathrm{L})\end{array}$ & $\begin{array}{c}\text { Capa- } \\
\text { cidad } \\
\text { de ad- } \\
\text { sorción } \\
(\mathrm{mg} / \mathrm{g})\end{array}$ \\
\hline CA6-15 & 0.0023 & 0.450 & 15 & 5.700 & 0.285 & 2.354 \\
\hline CA6-30 & 0.0023 & 0.450 & 30 & 5.700 & 0.329 & 2.335 \\
\hline CA6-60 & 0.0023 & 0.450 & 60 & 5.700 & 0.249 & 2.370 \\
\hline CA6-75 & 0.0023 & 0.450 & 75 & 5.700 & 0.254 & 2.368 \\
\hline CA6-90 & 0.0023 & 0.450 & 90 & 5.700 & 0.243 & 2.373 \\
\hline
\end{tabular}

Fuente: NSF Envirolab S.A.C (2017)

Considerando esas características del adsorbente, se evalúa el efecto de la dosis de adsorbente, en la remoción de Cd(II) de la solución preparada, independientemente de las otras variables, la dosis de adsorbente se establece en $0.0023 \mathrm{~g} / \mathrm{mL}$, un incremento de masa de adsorbente es incensario, ya que no influiría significativamente en la adsorción del Cd(II), debido a las interacciones electrostáticas entre las células del adsorbente, formando aglomerados que, por efecto de pantalla, disminuyen el número de centros activos disponibles para el Cd(II). Además, un incremento del estiércol de ganado vacuno tiende a secuestrar gran cantidad protones del medio elevando, en gran medida, el pH de la solución lo que comprometería la solubilidad del Cd(II).

\section{Conclusiones}

- El pH influye en los procesos de adsorción en dos sentidos diferentes: (1) alterando la solubilidad y distribución de las especies del Cd(II) en la disolución, (2) modificando el número de enlaces disponibles en la superficie del estiércol de ganado vacuno que se protonan o se desprotonan en función del $\mathrm{pH}$, esto es debido a que los protones presentes en el medio se unen fuertemente a los grupos funcionales del estiércol de ganado vacuno, principalmente carboxilos y fenoxidos, impidiendo el anclaje de los iones de $\mathrm{Cd}(\mathrm{II})$ por inhibición competitiva. Así mismo, los protones unidos al estiércol de ganado vacuno le confieren una carga neta positiva provocando fuerzas de repulsión que afectarían a la unión con los iones de $\mathrm{Cd}(\mathrm{II})$. El pH óptimo de adsorción de Cd(II) es 3,5; lo que implica, que en medio ácido se logra una desprotonación de la mayoría de los grupos funcionales del estiércol de ganado vacuno, se pueden desprotonar en iones carboxilato y fenoxido, con uno y dos átomos de oxígeno cargados negativamente, que actúan como agentes acomplejantes, los cuales atraen a los iones de $\mathrm{Cd}(\mathrm{II})$. Valores más altos de $\mathrm{pH}$ favorecen la formación de otros iones más complejos del $\mathrm{Cd}(\mathrm{II})$, como el ( $\mathrm{Cd}+2 \mathrm{OH}-)$, que dificultan la adsorción por su mayor volumen y su menor carga del Cd(II) en la solución.

- En cuanto a las isotermas de adsorción de Cd(II), su comportamiento lineal se debe a que la cantidad adsorbida de Cd(II) se incrementa conforme aumenta la concentración de $\mathrm{Cd}$ (II) en la solución preparada, esto indica que al incrementar la concentración de las soluciones de Cd(II), existirá un número mayor de iones de $\mathrm{Cd}$ (II) que se unirán a los centros activos, aumentando, así, las fuerzas de atracción y fortaleciendo la unión de más iones $\mathrm{Cd}(\mathrm{II})$ en dichos centros activos. La isoterma de adsorción lineal del tipo $\mathrm{C}$, indica que el adsorbato presenta mayor afinidad por al adsorbente que por el disolvente y que la isoterma indica que el soluto penetra en zonas inaccesibles al disolvente. La isoterma lineal del subgrupo 1 representa que la monocapa no ha sido completada por los adsorbatos.

- Finalmente, se ha encontrado que la capacidad de adsorción del estiércol de ganado vacuno, es proporcional a la concentración de Cd(II) presente en la solución y al tiempo de contacto, para un $\mathrm{pH}$ de 3,5; esto indica que con $1 \mathrm{~g}$ de adsorbente se adsorbe 2,3726 mg de Cd(II). 


\section{Referencias bibliográficas}

Atusta Rengifo CH. Comparación de cinco métodos para determinar capacidad de intercambio catiónico en suelos alcalinos. Colombia: CXorporación Autónoma Regional del Cauca, Departamento Agropecuario-Sección de Suelos.

Caballero Caballero E. "XVI Verano de la investigación científica”. In Ramón Frías T, editor. Memorias del XVIVerano de la investigación científica de la UJAT 2006; 2006; Tabasco México: Universidad Juárez Autónoma de Tabasco. p. 109-110.

Chuquilín Goicochea RC, Rosales Laguna DD. Estudio de la biosorción de $\mathrm{Cd}$ (II) y $\mathrm{Pb}$ (II) usando como adsorbente Nostoc sphaericum Vaucher. Revista de la Sociedad Química del Perú. 2016 Marzo 30; 80(1): p.

Marín Martínez AJ. Estudio de adsorción de boro con amberlite IRA 743. Tesis de grado. Barcelona España: Universidad Potitécnica de Catalunya de España, Departamento 713 Ingeniería Química; 2011.

Mejía Sandoval G. "Aproximación teórica a la biosorción de metales pesados por medio de microorganismos”. Revista CES Medicina Veterinaria y Zootecnia. 2006 Junio; I(1): p. 89.

Ramos Yánac KP, Llanos Chang BP, Maldonado García HJ, Navarro Aliaga AE. "Evidencias del mecanismo de adsorción del cadmio divalente en Lentinus edodes”. Revista Real Sociedad Española de Química. 2007; 103: p. 36.

Rosas R. H. Estudio de la contaminación por metales pesados en la cuenca del Llobregat. Universitat Politècnica de Catalunya. 2001. ISBN: 8469956558. Recuperado de: http: / /hdl.handle.net/10803/6978

Soto Huaringa E. "Estudio de la remoción de Cu(II) en medio acuoso utilizando el albedo de las cáscaras de naranja”. Tesis de grado. Lima Perú: Universidad Nacional de Ingeniería, Facultad de Ciencias; 2009.

Ures Rodríguez P, Suárez López J, Jácome Burgos A. "Adsorción en carbón activo (FT-TER-002)". Fichas técnicas de etapas de procesos de plantas de tratamiento de agua residuales de la industria textil. 2015 Mayo; 002: p. 5-6.

Vullo DL. "Microorganismos y metales pesados: Una interacción en beneficio del medio ambiente". Revista Química Viva, Buenos Aires Argentina. 2003 Noviembre $12 ; 2(3)$ : p. 1-2. 